\title{
LE FONCTIONNEMENT D'UN SITE DE TRANSMISSION A SCHISTOSOMA MANSONI EN MARTINIQUE (ANTILLES FRANÇAISES)
}

\author{
J.-P. POINTIER*, A. GUYARD**, A. THERON*** et A. DUMOUTIER****
}

RÉSUMÉ. Un foyer de bilharziose intestinale a été découvert en novembre 1981 dans la région de Saint-Pierre en Martinique. Une analyse de sa structure et du mécanisme de la transmission parasitaire a été entreprise entre 1981 et 1983. Ce foyer présente les particularités suivantes : un site de transmission très localisé constitué par des cressonnières ; une petite population humaine présentant un taux d'infestation de $13 \%$ (coprologies) à $41,3 \%$ (sérologies) ; une contamination fécale des eaux faible, ponctuelle et régulière représentée par le déversoir d'un bassin d'épuration non fonctionnel ; une forte population du Mollusque vecteur Biomphalaria glabrata $(40,5$ à 256,3 invidivus $\left./ \mathrm{m}^{2}\right)$; une faible prévalence d'infestation des Mollusques $(0,13$ à $0,59 \%)$; de faibles densités cercariennes dans les eaux des cressonnières (0,1 à 1,2 cercaires par litre) ; et enfin, une très faible contamination des eaux de la rivière dans laquelle aboutissent les eaux des cressonnières (la Roxelane). L'importance épidémiologique de cette contamination s'est révélée faible mais pas nulle. Ce foyer apparaît comme un cas particulier en Martinique car toutes les prospections réalisées à ce jour dans l'île n'ont pas permis de retrouver un tel exemple.

\section{Studies on a schistosomiasis mansoni focus in Martinique (French West Indies)}

SUMMARY. A focus of intestinal schistosomiasis was discovered in november 1981 in Martinique in the Saint-Pierre area. An analysis of its structure and of the mecanisms of the transmission of the parasite was carried out between 1981 and 1983. This focus presents the following particularities : a transmission site very small constituted by water cress beds; a small human population infected with a prevalence of $13 \%$ (positive stools) to $41,3 \%$ (positive serologies) ; a weak fecal contamination of the waters; a non functionning sewage stabilization tank is responsible for this contamination ; a rich population of the snail host Biomphalaria glabrata $(40,5$ to $\left.256,3 \mathrm{Bg} / \mathrm{m}^{2}\right)$ with a low prevalence of infestation $(0,13$ to $0,59 \%)$; low cercarial densities in the water eress bed waters ; a very low contamination of the Roxelane river water in to which the water from the water cress beds flows. The epidemiological importance of this contamination has proved very low but not altogether absent. This focus appears as a particular case in Martinique because all the surveys which have been conducted in the island to date has not uncovered a similar example.

* Laboratoire de Biologie marine et Malacologie, École Pratique des Hautes Études, 55 rue Buffon, F 75005 Paris.

** Laboratoire de Biologie animale, Université Antilles-Guyane, BP 592, 97167 Pointe-à-Pitre, Guadeloupe (FWI).

*** Département de Biologie animale, Université de Perpignan, Avenue-de-Villeneuve, F 66025 Perpignan.

**** Service Parasitoses, D.D.A.S.S., Boulevard Pasteur, 972 Fort-de-France, Martinique (FWI).

Accepté le 9 janvier I984. 


\section{Introduction}

En Martinique, la bilharziose intestinale fut mise en évidence en début du siècle mais une étude générale de sa fréquence et de sa répartition ne fut réalisée qu'en 1977 par l'Unité 165 de l'INSERM. Cette enquête a montré que la prévalence moyenne de la maladie chez l'homme est de $12 \%$ pour l'ensemble de l'île (sérologie et selles confondues). La zone la plus touchée est le Nord avec des prévalences de plus de $40 \%$ pour la région de Saint-Pierre.

Depuis quelques années nous avons entrepris des études sur la répartition du Mollusque vecteur (Guyard et Pointier, 1979) ainsi que sur la transmission parasitaire (Guyard et al., 1982-1983a, Pointier, 1982). Toutes ces études tendent à montrer que la transmission de la bilharziose intestinale en Martinique est aujourd'hui presque totalement interrompue. Cette interruption, vraisemblablement progressive, remonte à plus d'une dizaine d'années comme le suggère l'absence d'infestation des tranches d'âge les plus jeunes de la population humaine (Anonyme, 1979, Guyard et al., 1983b).

Plusieurs facteurs semblent responsables de cette interruption : forte régression du Mollusque vecteur Biomphalaria glabrata à la suite de la colonisation des collections d'eau martiniquaises par B. straminea ; diminution de la contamination fécale des eaux consécutive au développement des adductions d'eau et des installations sanitaires ; modification du comportement humain vis-à-vis de l'eau.

Cependant, en novembre 1981, un site de transmission fonctionnel a été découvert à Saint-Pierre dans le quartier du Jardin des Plantes. Une analyse de sa structure et des mécanismes de la transmission parasitaire a donc été entreprise dans ce foyer particulier.

\section{Présentation du foyer}

Ce foyer comprend un quartier d'habitations récentes. Le lotissement comporte actuellement 26 maisons dont certaines sont encore en cours de construction. Toutes ces habitations sont dotées d'eau courante et d'installations sanitaires dont le réseau d'évacuation aboutit à un bassin d'épuration situé en bordure de la rivière du Jardin des Plantes. Ce bassin n'a jamais été fonctionnel et le trop plein se déverse dans la rivière peu avant le confluent rive gauche de la rivière Roxelane. Une cinquantaine de mètres en aval, une prise d'eau sur la Roxelane, qui recueille une grande partie de l'eau provenant de la rivière du Jardin des Plantes, alimente deux groupes de cressonnières qui hébergent les populations de Mollusques vecteurs. Ces cressonnières constituent donc le site de transmission du foyer. Après avoir irrigué les cressonnières, l'eau retourne à la Roxelane en aval (fig. 1).

\section{La parasitose chez l'homme}

Un dépistage systématique a été effectué par la DDASS début 1983 et a porté sur la quasi totalité de la population du quartier (46 personnes examinées sur 49). 


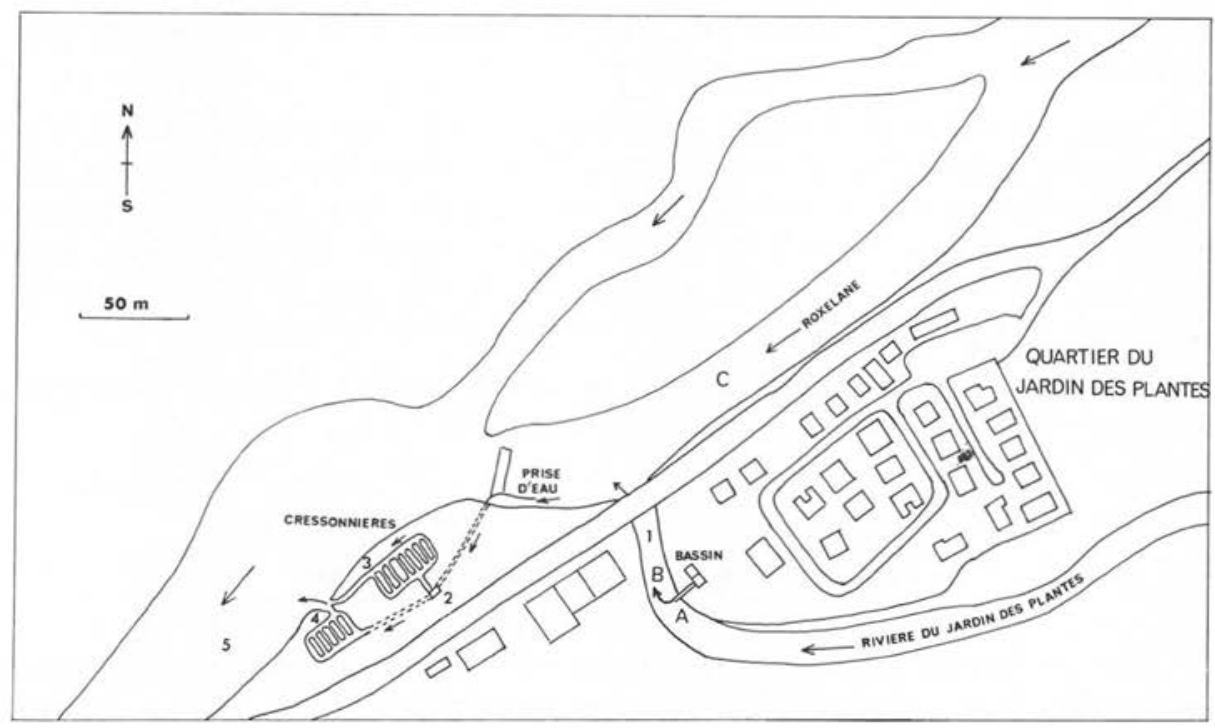

FIg. I. - Le foyer du quartier du Jardin des Plantes à Saint-Pierre. Situation du point de contamination (bassin), du site de transmission (cressonnières), des points de filtration (n ${ }^{\text {os }}$ I à 5) et des emplacements des cages contenant les Mollusques sentinelles (points A-B-C).

Les analyses de selles ont été réalisées par la méthode de Bailenger et Janeckso et les sérologies par hémagglutination passive.

Les résultats montrent une prévalence de $41,3 \%$ pour les sérologies (soit 19 personnes positives à un seuil de $\left.1 / 40^{\mathrm{e}}\right)$ et de $13 \%$ pour les selles ( 6 personnes positives). L'examen de la répartition de la parasitose par tranches d'âge montre l'absence d'infestation chez les enfants de moins de 10 ans, tant du point de vue sérologique que coprologique.

Des œufs de Schistosoma mansoni sont donc déversés régulièrement dans la rivière du Jardin des Plantes par l'intermédiaire du bassin d'épuration.

\section{La contamination fécale de la rivière}

Afin de préciser l'origine du (ou des) points de contamination des planorbes et d'estimer son importance, des cages contenant des Mollusques sentinelles (des B. glabrata nés et élevés au laboratoire) ont été placées en trois points de la rivière du Jardin des Plantes et de la Roxelane (fig. 1, points A-B-C) :

- la première dans la rivière du Jardin des Plantes avant le déversoir du bassin d'épuration (A) ;

- la deuxième dans la rivière du Jardin des Plantes après le déversoir du bassin d'épuration (B) ; 
- la troisième dans la Roxelane en amont du confluent de la rivière du Jardin des Plantes (C).

Après un séjour de 72 heures dans l'eau, les trois cages ont été retirées et les Mollusques placés en élevage au laboratoire. Les résultats des tests d'émission cercariennes effectués après la période nécessaire à la maturation des parasites, ont été négatifs en amont du déversoir du bassin d'épuration (point A), négatifs également dans la Roxelane (point $\mathrm{C}$ ), mais positifs après le déversoir du bassin (point $\mathrm{B}$ ). A cet endroit, le taux d'infestation des Mollusques sentinelles a été de 1,5\%.

Ces résultats démontrent clairement le rôle du déversoir du bassin d'épuration dans la contamination des planorbes des cressonnières.

\section{Le mollusque hôte intermédiaire}

Les Mollusques sont présents uniquement dans les cressonnières. Celles-ci hébergent de riches populations de Biomphalaria glabrata.

Trois estimations de la densité des Mollusques ont été effectuées par la méthode des quadrats en novembre 1981, en février 1983 et en novembre 1983. Tous les Mollusques repérés dans les carrés de $1 / 20 \mathrm{~m}^{2}$ régulièrement espacés sont prélevés à la pince, comptés et testés quant à l'émission éventuelle de cercaires de Schistosoma mansoni. Un total de 150 échantillons de $1 / 20 \mathrm{~m}^{2}$ ont été ainsi récoltés dans les deux groupes de cressonnières. Les résultats rapportés à la superficie totale des cressonnières ont permis d'évaluer la densité $/ \mathrm{m}^{2}$ et le nombre total de Mollusques sains et parasités. Les résultats présentés dans le tableau $I$ amènent les remarques suivantes :

- les populations de $B$. glabrata $(\mathrm{Bg})$ des deux groupes de cressonnières ont des densités élevées qui peuvent varier d'une année à l'autre dans de fortes proportions : 49,5 à $152,7 \mathrm{Bg} / \mathrm{m}^{2}$ dans le premier groupe de cressonnières et 40,5 à $256,3 \mathrm{Bg} / \mathrm{m}^{2}$ dans le deuxième groupe ;

- les prévalences d'infestation par S. mansoni sont faibles $(0,13$ à $0,59 \%)$ et s'expliquent par l'éloignement du point de rejet fécal qui est de l'ordre de 200 mètres (fig. 1);

- en novembre 1983, aucun Mollusque infesté n'a pu être récolté. Ce résultat est vraisemblablement la conséquence de la chimiothérapie des malades qui avaiert été dépistés 9 mois plus tôt, en février 1983. Ce traitement a donc interrompu l'apport d'œufs de parasites dans la rivière alimentant les cressonnières ;

- une deuxième espèce de planorbe, Biomphalaria straminea (Bs), est également présente dans les cressonnières mais en plus faibles densités que B. glabrata. Ces densités ont varié de 1,7 à $29,8 \mathrm{Bs} / \mathrm{m}^{2}$ dans le premier groupe de cressonnières et de 2,3 à $11,9 \mathrm{Bs} / \mathrm{m}^{2}$ dans le deuxième groupe. Aucun $B$. straminea infesté n'a pu être récolté entre 1981 et 1983. 
TABleau I. - Caractéristiques des propulations de Biomphalaria glabrata $(\mathrm{Bg})$ présentes dans les deux groupes de cressonnières entre 1981 et 1983. On remarquera l'absence de Mollusques infestés $\left(\mathrm{Bg}^{+}\right)$par Schistosoma mansoni dans les prélèvements effectués en novembre 1983.

\begin{tabular}{lccc}
\hline Cressonnière 1 & Novembre 1981 & Février 1983 & Novembre 1983 \\
\hline $\mathrm{Bg} / \mathrm{m}^{2}$ & $49,2 \pm 23,2$ & $131,5 \pm 41,7$ & $152,7 \pm 31,3$ \\
$\mathrm{~N}$ Total & $26.024 \pm 12.270$ & $69.589 \pm 22.084$ & $80.799 \pm 16.584$ \\
$\mathrm{Bg}+\mathrm{m}^{2}$ & $0,29 \pm 0,13$ & $0,27 \pm 0,09$ & 0 \\
$\mathrm{~N}$ Total & $153 \pm 72$ & $146 \pm 46$ & 0 \\
Prévalence & $0,59 \%$ & $0,21 \%$ & 0 \\
moyenne & Novembre 1981 & Février 1983 & Novembre 1983 \\
\hline \multicolumn{1}{c}{ Cressonnière 2} & $82,3 \pm 16,5$ & $256,3 \pm 81,1$ & $40,5 \pm 15,3$ \\
Bg/m ${ }^{2}$ & $36.556 \pm 9.133$ & $113.811 \pm 35.199$ & $12.117 \pm 4.593$ \\
$\mathrm{~N}$ Total & $0,28 \pm 0,07$ & $0,33 \pm 0,10$ & 0 \\
Bg $/ \mathrm{m}^{2}$ & $128 \pm 32$ & $148 \pm 47$ & 0 \\
$\mathrm{~N}$ Total & $0,35 \%$ & $0,13 \%$ & 0 \\
Prévalence & & & \\
moyenne & & & \\
\hline
\end{tabular}

\section{Les densités cercariennes dans les eaux}

Des échantillons d'eau ont été prélevés à différentes périodes en cinq points du réseau hydrographique ( $f i g .1$, points $\mathrm{n}^{\mathrm{os}} 1$ à 5 ) : $\mathrm{n}^{0} 1$ au confluent de la rivière du Jardin des Plantes, n 2 au niveau du canal d'amenée d'eau aux cressonnières, no 3 à l'effluent du premier groupe de cressonnières, no 4 à l'effluent du deuxième groupe de cressonnières, no 5 dans la Roxelane en aval des cressonnières. Les échantillons d'eau ont été ensuite ramenés au laboratoire, filtrés, et les cercaires de $S$. mansoni dénombrées selon la technique de Theron (1979).

Les résultats sont négatifs au confluent de la rivière du Jardin des Plantes (point $\mathrm{n}^{\circ} 1$ ) ainsi qu'au niveau du canal d'amenée d'eau aux cressonnières (point $\mathrm{n}^{\circ} 2$ ). En aval des cressonnières (points n ${ }^{\text {os }} 3$ et 4), on trouve de 0,1 à 1,2 cercaires par litre d'eau selon le point et la période de prélèvement (tableau II).

Dans la Roxelane, en aval des cressonnières (point no 5), les résultats sont négatifs malgré l'apport de celles-ci. Cela s'explique par la forte dilution causée par le débit de la Roxelane qui est de l'ordre de $1 \mathrm{~m} 3 / \mathrm{s}$ alors que celui du canal de sortie des cressonnières est de l'ordre de $0,02 \mathrm{~m} 3 / \mathrm{s}$. Les densités cercariennes de la Roxelane sont 
TABLEAU II. - Taux cercariens (cercaires par litre d'eau) mesurés dans les eaux du foyer du Jardin des Plantes. On se reportera à la carte Fig. 1 pour l'emplacement des 5 points de filtration.

\begin{tabular}{cccccc}
\hline $\begin{array}{c}\text { Points de } \\
\text { filtration }\end{array}$ & $\begin{array}{c}\text { Novembre } \\
1981\end{array}$ & $\begin{array}{c}\text { Avril } \\
1982\end{array}$ & $\begin{array}{c}\text { Novembre } \\
1982\end{array}$ & $\begin{array}{c}\text { Février } \\
1983\end{array}$ & $\begin{array}{c}\text { Novembre } \\
1983\end{array}$ \\
\hline 1 & 0 & 0 & 0 & 0 & 0 \\
2 & 0 & 0 & 0 & 0 & 0 \\
3 & $1,2 \mathrm{c} / 1$ & $0,4 \mathrm{c} / 1$ & $1,2 \mathrm{c} / 1$ & $0,6 \mathrm{c} / 1$ & 0 \\
4 & $0,4 \mathrm{c} / 1$ & $0,1 \mathrm{c} / 1$ & $0,2 \mathrm{c} / 1$ & $0,3 \mathrm{c} / 1$ & 0 \\
5 & 0 & 0 & 0 & 0 & 0 \\
\hline
\end{tabular}

donc beaucoup trop faibles (inférieures à $0,02 \mathrm{c} / 1$ par le jeu de la dilution) pour être détectées par la méthode utilisée à moins de filtrer des volumes d'eau très importants. Quoi qu'il en soit, ces chiffres suffisent à montrer que les risques de contamination humaine sont très faibles dans la Roxelane. Ils ne sont cependant pas nuls et peuvent sans doute expliquer les résultats d'un dépistage qui a été effectué au niveau scolaire à Saint-Pierre, et qui a permis de détecter deux enfants âgés de trois et quatre ans porteurs d'œufs de $S$. mansoni. Ces enfants auraient fréquenté en été 1981 les eaux de la Roxelane, probablement en étiage, au niveau de l'embouchure, soit un kilomètre environ en aval des cressonnières contaminées.

Dans les cressonnières elles-mêmes, les risques de contamination sont accrus avec des taux de 0,1 à $1,2 \mathrm{c} / 1$ qui se révèlent relativement constants au cours de la période de novembre 1981 à février 1983 (tableau II). Il convient toutefois de signaler l'échantillonnage négatif de novembre 1983 qui confirme l'absence de Mollusques infestés dans les prélèvements de cette période et l'interruption de la contamination fécale.

\section{Conclusions}

Ce foyer du quartier du Jardin des Plantes présente les particularités suivantes : — un site de transmission très localisé constitué par des cressonnières ;

- une petite population humaine infestée à un taux non négligeable $(41,3 \%$ de sérologies et $13 \%$ de selles positives) ;

- une contamination fécale des eaux faible, ponctuelle et régulière représentée par le déversoir d'un bassin d'épuration non fonctionnel ;

- une forte population du Mollusque vecteur Biomphalaria glabrata présentant une faible prévalence parasitaire $(0,13$ à $0,59 \%)$; 
- de faibles densités cercariennes dans les eaux des cressonnières qui confirment la faible prévalence d'infestation des Mollusques $(0,1$ à 1,2 c/1) ;

- une très faible contamination des eaux de la Roxelane par les cressonnières dont l'importance épidémiologique s'est révélée faible mais pas nulle.

Le dépistage et le traitement par l'oxamniquine des sujets contaminés a apparemment suffit à rompre le cycle parasitaire dans ce petit foyer. Il convient toutefois de signaler que seul un concours de circonstances peu ordinaire avait permis son installation : bassin d'épuration non fonctionnel situé juste en amont d'un groupe de cressonnières hébergeant de riches populations du Mollusque vecteur. Toutes les prospections réalisées à ce jour en Martinique n'ont pas permis de retrouver un tel exemple. Malgré son caractère très particulier, une telle situation montre la nécessité d'intégrer des programmes de surveillance des collections d'eau dans les plans de développements des départements d'Outre-Mer.

\section{BIBLIOGRAPHIE}

ANONYME : Étude de l'endémie parasitaire intestinale dans les départements d'Outre-Mer. I. - La Martinique. U I65-INSERM, Le Vésinet I979, $5^{8}$ p.

GuYARD A., PoIntier J. P. : Faune malacologique dulçaquicole et vecteurs de la schistosomose intestinale en Martinique (Antilles françaises). Ann. Parasitol. Hum. Comp., 1979, 54, 193-205.

Guyard A., Pointier J. P., Theron A., Gilles A. : Mollusques hôtes intermédiaires de la schistosomose intestinale dans les Petites Antilles : hypothèses sur le rôle de Biomphalaria glabrata et B. straminea en Martinique. Malacologia, 1982, 22, 103-107.

Guyard A., Pointier J. P., Theron A. : The decline of Schistosoma mansoni in Martinique (French West Indies). Role of the competitive displacement of the snail host Biomphalaria glabrata by B. straminea Eight. Int. Mal. Cong. Budapest 28 aug. - 2 sept. 1983. Abstr., I983a, 49.

Guyard A., Pointier J. P., Theron A., Dumoutier A. : La bilharziose intestinale en Martinique, en 1983 . Recensement et fonctionnement des sites de transmission. CRDP de Fort-deFrance, $1983 \mathrm{~b}, 25 \mathrm{p}$.

Pornter J. P. : Possible competitive displacement of Biomphalaria glabrata (intermediate host snail of schistosomiasis) by B. straminea in Martinique (French West Indies). Fifth Int. Cong. Parasit. Toronto 7-I4 Aug. 1982. Abstr., 1982, 429-430.

Theron A. : A differential filtration technique for the measurement of schistosome cercarial densities in standing waters. Bull. Wld. Hlth. Org., 1979, 57, 971-975. 